\title{
NUEVO REGISTRO DE PSILOCYBE (FUNGI, BASIDIOMYCOTINA, AGARICALES) EN ARGENTINA
}

\author{
Alejandro José Moyano y Graciela María Daniele \\ Instituto Multidisciplinario de Biología Vegetal (IMBIV)-CONICET \\ Universidad Nacional de Córdoba \\ Casilla de correo 495, 5000 Córdoba, Argentina \\ ajmoyano@hotmail.com
}

\section{RESUMEN}

Se amplía la distribución y el sustrato conocidos de Psilocybe pegleriana. Éste constituye el registro más austral en América para la especie y es la primera cita para la Argentina.

Palabras clave: Argentina, Psilocybe, Sudamérica.

\section{ABSTRACT}

An extension of the known distribution and substrate is reported for Psilocybe pegleriana. This constitutes the most austral record of the species for America and it is the first record for Argentina.

Key words: Argentina, Psilocybe, South America.

\section{INTRODUCCIÓN}

En Argentina el género Psilocybe fue citado en distintas oportunidades por Spegazzini (1887, 1922, 1923, 1926 a-b), Singer (1950, 1959 a-b), Singer y Digilio (1951), Horak (1967, 1979), Guzmán y Horak (1978), Guzmán (1983) y Raithelhuber (1988, 1991).

En la provincia de Córdoba, ubicada en la zona central del país, los registros relacionados con la diversidad fúngica son escasos, lo cual no es ajeno a este género. Sólo han sido citadas previamente $P$. jujuyensis Singer (Raithelhuber, 1991) (especie dudosa según observaciones de Guzmán, 1983) y Deconica coprophila Bull. [= Psilocybe coprophila (Bull.: Fr.) P. Kumm.] (Spegazzini, 1926 a). Esta última merece ser revisada, ya que Spegazzini $(1887,1923)$ identificó colecciones de la provincia de Tierra del Fuego como Agaricus coprophilus Bull. y Deconica coprophila, las que luego fueron redeterminadas por Horak $(1967,1979)$ como $D$. fuegiana Horak [= Psilocybe fuegiana (Horak) Singer.] y D. subcoprophila Britzelm. 
(= Psilocybe subcoprophila (Britzelm.) Sacc.) respectivamente. Tanto D. fuegiana como D. subcoprophila, fueron discutidas por Guzmán (1983).

En ocasión de explorar la micobiota agaricina de ambientes pratícolas de la provincia de Córdoba, Argentina, se recolectaron basidiomas de una especie del género Psilocybe no citada aún para Argentina, hecho que constituye la base del presente trabajo.

\section{MATERIALES Y MÉTODOS}

Los basidiomas fueron estudiados macroscópicamente en fresco. Para el estudio de las estructuras microscópicas se realizaron cortes a mano montados en $\mathrm{KOH}$ a 5\%, floxina y agua siguiendo los lineamientos de Largent et al. (1977). Los dibujos de dichas estructuras fueron realizados con el auxilio de una cámara clara. El material utilizado en este estudio se encuentra depositado en el Herbario del Museo Botánico de Córdoba (CORD).

\section{RESULTADOS}

Psilocybe pegleriana Guzmán, Documents Mycologiques 29: 43 (2000).

$=$ Psilocybe pseudobullacea (Petch) Pegler, sensu Pegler, Kew Bull. Add. Ser. 6: 473 (1977); sensu Guzmán, Beit. Nov. Hedw. 74: 240 (1983).

= Psilocybe merdaria (Fr.) Ricken sensu Guzmán, Bol. Soc. Mex. Mic. 11: 30 (1977). Fig. 1

Píleo de 5-15 mm de diámetro, convexo o cónico-acampanado cuando joven a levemente aplanado en la madurez, subumbonado, superficie glabra, higrófana y levemente viscosa cuando húmeda, de color crema claro en el margen a cremaanaranjado o pardo en el centro, margen apenas estriado, con reborde blanquecino correspondiente a restos del velo. Láminas adnatas con un diente decurrente, distantes, de color marrón, tornándose violáceas oscuras; aristas blanquecinas muy notables. Estípite de 30-70 x 1-2.5 mm, central, isodiamétrico en toda su longitud, en ocasiones un poco más ancho en su extremo superior; fistuloso, de color pardo claro, más claro en el tercio superior, con micelio blanco en la base. Anillo membranoso, estriado en su cara superior y flocoso en la inferior. Contexto escaso, blanquecino. Esporas de 9.5-11.2 × 6.4-8 $\mu \mathrm{m}$, cara frontal subhexagonal, cara lateral elipsoidal; pared gruesa (ca. $1 \mu \mathrm{m}$ ), pardas, con poro germinal apical. Basidios de 18-21 x 7.8-9 $\mu \mathrm{m}$, claviformes, tetrasporados; esterigmas de 3-5 $\mu \mathrm{m}$. Pleurocistidios ausentes. Queilocistidios de 19-27.5 x 4.8-6.5 $\mu \mathrm{m}$, sublageniformes, hialinos, de pared delgada, frecuentemente con una gota apical refringente. Trama del himenóforo regular. Pileipelis subgelatinosa compuesta por hifas hialinas de 2-4.8 $\mu \mathrm{m}$ de diámetro. Fíbulas presentes. 
Moyano y Daniele: Nuevo registro de Psilocybe en Argentina
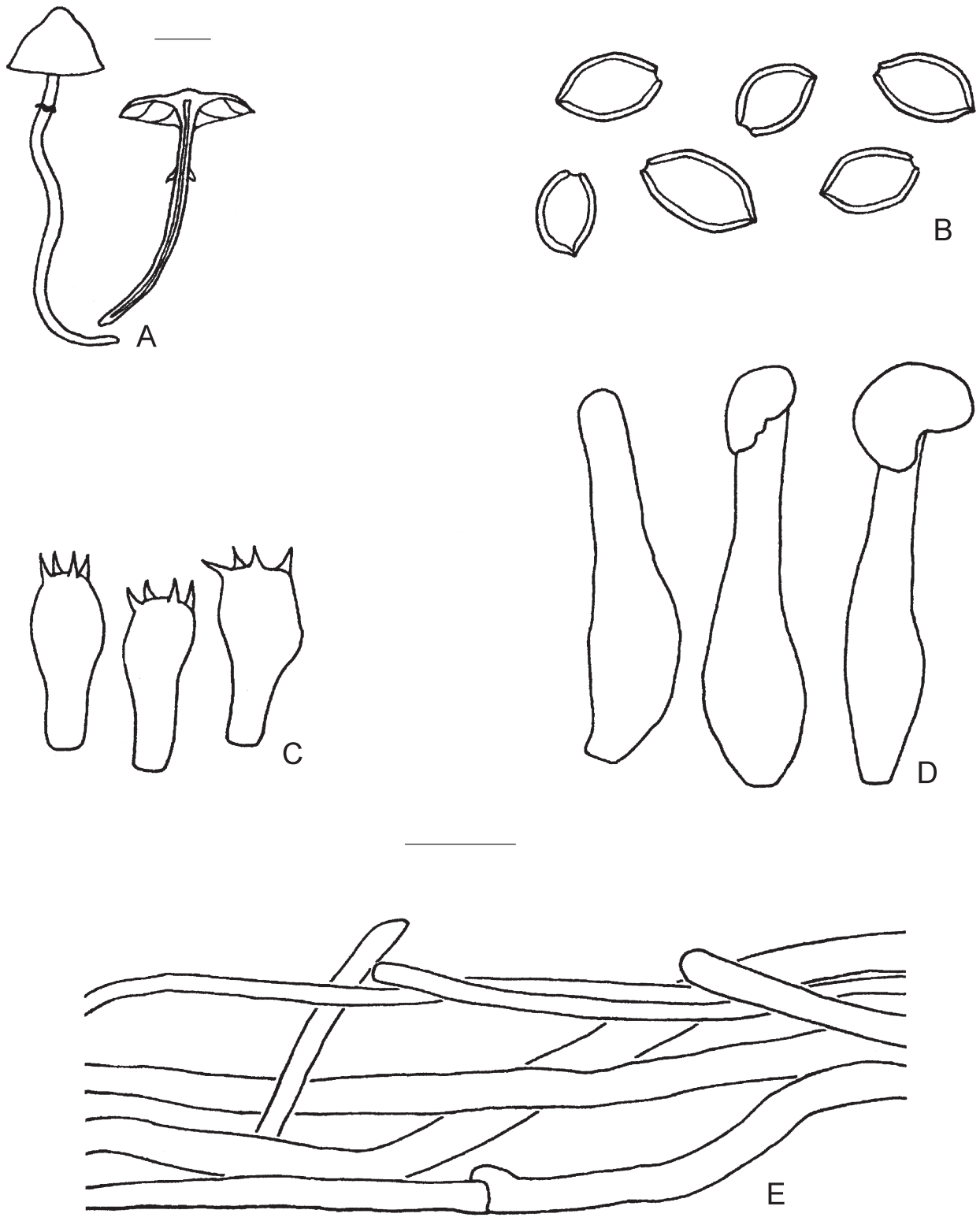

Fig. 1. Psilocybe pegleriana. A. basidiomas; B. esporas; C. basidios; D. queilocistidios; E. pileipelis. Escala $4 \mathrm{~mm}$ en $\mathrm{A} ; 11 \mathrm{~mm}$ en B-E.

27 
Hábitat y distribución: Si bien Guzmán (2000) citó esta especie creciendo en restos de caña de azúcar, el material estudiado se recolectó en restos de plantas de maíz (Zea mays L.) trituradas y almacenadas en silos embutidos como reserva forrajera. En el mismo sustrato se encontraron basidiomas de otros taxa pertenecientes a los géneros Coprinus, Panaeolus, Psilocybe, Agrocybe, Bolbitius y Conocybe.

$P$. pegleriana fue citada en dos oportunidades bajo el nombre de $P$. pseudobullacea en Ecuador y Venezuela (Guzmán, 1983, 2000); ambos registros necesitan ser revisados, según Guzmán (2000). Hasta ahora, Ecuador constituía el límite austral de distribución de $P$. pegleriana en América (Guzmán, 2000), por lo tanto este hallazgo amplía en gran medida su área conocida de distribución.

Material estudiado: Argentina, Prov. Córdoba, Dpto. Colón, km 730 ruta nacional $n^{\circ}$ 9, 16.III.2000, A. Moyano 6 (CORD); ibidem 21.V.2000, A. Moyano 39 (CORD); ibidem 02.XI.2000, A. Moyano 46 (CORD, XAL).

\section{AGRADECIMIENTOS}

Queremos expresar nuestro agradecimiento al Profesor Dr. Gastón Guzmán, quien actualizó la determinación del material en cuanto a su nomenclatura; a la Dra. Laura Domínguez, Biól. Carlos Urcelay y Biól. Gerardo L. Robledo por la lectura crítica del manuscrito.

El presente trabajo ha sido subsidiado por la Agencia Córdoba Ciencia (Expte. 01130200151 1) y por SECYT (Res. 194100).

\section{LITERATURA CITADA}

Guzmán, G. 1983. The genus Psilocybe. Beih. Nova Hedwigia. 74. J. Cramer. Vaduz. 479 pp. Guzmán, G. 2000. New species and new records of Psilocybe from Spain, the U.S.A. and Mexico, and a new case of poisoning by Psilocybe barreras. Documents Mycologiques 29(116): 41-52.

Guzmán, G. y E. Horak. 1978. A new species of Psilocybe (belonging to the P. crobulagroup) from Argentina. Mycotaxon 7: 521-522.

Horak, E. 1967. Fungi austroamericani IV. Revisión de los hongos superiores de Tierra del Fuego o Patagonia en el herbario de C. Spegazzini en La Plata. Darwiniana 14: 355385.

Horak, E. 1979. Fungi Basidiomycetes. Agaricales y Gasteromycetes Secotioides. In Guarrera S. A., Gamundi de Amos I. y Rabinovich de Halperin D. (eds.). Flora criptogámica de Tierra del Fuego. CONICET-FECIC. Buenos Aires. 528 pp.

Largent, D., D. Johnson y R. Watling. 1977. How to identify mushrooms to genus III: Microscopic features. Mad River Press. Eureka, California. 148 pp.

Raithelhuber, J. 1988. Flora mycologica argentina; Hongos II. Ed. Mycosur. Stuttgart. 287 pp. 
Raithelhuber, J. 1991. Flora mycologica argentina; Hongos III. Ed. Mycosur. Stuttgart. 500 pp.

Singer, R. 1950. Type studies on Basidiomycetes IV. Lilloa 23: 147-246.

Singer, R. 1959a. Hongos alucinógenos. Bol. Acad. Nac. Cienc. Córdoba 41: 31-46.

Singer, R. 1959b. New and interesting species of Basidiomycetes VII. Mycologia 51: 578594.

Singer, R. y A. P. L. Digilio. 1951. Pródromo de la flora agaricina argentina. Lilloa 25: 5461.

Spegazzini, C. 1887. Fungi Fuegiani. Bol. Acad. Nac. Cienc. Córdoba 11: 135-308.

Spegazzini, C. 1922. Cryptogamae nonnullae Fuegianae. Resultado de la primera expedición a Tierra del Fuego. An. Soc. Cient. Argent. 94: 59-85.

Spegazzini, C. 1923. Relación de un paseo hasta el Cabo de Hornos. Bol. Acad. Nac. Cienc. Córdoba 27: 321-404.

Spegazzini, C. 1926a. Contribución al conocimiento de la flora micológica de las sierras de Córdoba. Bol. Acad. Nac. Cienc. Córdoba 29: 113-190.

Spegazzini, C. 1926b. Observaciones y adiciones a la micología argentina. Bol. Acad. Nac. Cienc. Córdoba 28: 267-351. 